\title{
The association between diabetes mellitus and reduction in myocardial glucose uptake: a population-based ${ }^{18} \mathrm{~F}$-FDG PET/CT study
}

\author{
Lijun $\mathrm{Hu}^{1}$, Chun Qiu' ${ }^{2}$ Xiaosong Wang ${ }^{2}$, Mei Xu' ${ }^{2}$ Xiaoliang Shao ${ }^{2^{*}}$ (D) and Yuetao Wang ${ }^{2^{*}}$
}

\begin{abstract}
Background: In diabetes, dysregulated substrate utilization and energy metabolism of myocardium can lead to heart failure. To examine the dynamic changes of myocardium, most of the previous studies conducted dynamic myocardial PET imaging following euglycemic-hyperinsulinemic clamp, which involves complicated procedures. In comparison, the whole-body ${ }^{18} \mathrm{~F}$-FDG PET/CT scan is a simple and widely used method. Therefore, we hope to use this method to observe abnormal myocardial glucose metabolism in diabetes and determine the influencing factors.

Methods: We retrospectively analyzed PET/CT images of 191 subjects from our medical examination center. The levels of FDG uptake in myocardium were visually divided into 4 grades (Grade $0-3$, from low to high). The differences in clinical and metabolic parameters among diabetes mellitus (DM), impaired fasting glucose (IFG), and normal fasting glucose (NFG) groups were analyzed, as well as their associations with myocardial FDG uptake.

Results: Compared with NFG and IFG groups, DM group had more cardiovascular-related risk factors. The degree of myocardial FDG uptake was significantly decreased in DM group; when myocardial FDG uptake $\leq$ Grade 1, the sensitivity of DM prediction was $84.0 \%$, and the specificity was $58.4 \%$. Univariate analysis showed that the myocardial FDG uptake was weakly and negatively correlated with multiple metabolic-related parameters $(r=-0.173 \sim-0.365, P<$ 0.05). Multivariate logistic regression analysis showed that gender (male), HOMA-IR and nonalcoholic fatty liver disease (NAFLD) were independent risk factors for poor myocardial FDG uptake.
\end{abstract}

Conclusions: Diabetes is associated with decreased myocardial glucose metabolism, which is mediated by multiple metabolic abnormalities.

Keywords: Diabetes mellitus, FDG, PET, Insulin resistance, Nonalcoholic fatty liver disease

\section{Background}

Free fatty acids and glucose are the major substrates for myocardium energy metabolism. The process of substrate selection is dynamic and largely depends on substrate availability, oxygen concentration, and myocardial workload [1]. Under pathological conditions (such as diabetes, myocardial ischemia, and left ventricular hypertrophy), the abnormal substrate utilization, energy metabolism disorder, mitochondria dysfunction, and ATP synthesis failure [2],

\footnotetext{
* Correspondence: xl_shao@126.com; yuetao-w@163.com

${ }^{2}$ Department of Nuclear Medicine, The Third Affiliated Hospital of Soochow University, Changzhou 213003, Jiangsu, China

Full list of author information is available at the end of the article
}

can all lead to myocardial structural changes and reduction in systolic/diastolic function, which then trigger heart failure. Therefore, there is an increasing demand for accurate and non-invasive imaging approaches to observe various aspects of myocardial substrate metabolism.

Diabetes is a metabolic disorder characterized by hyperglycemia, dyslipidemia and insulin resistance [3]. Diabetic patients are at higher risks of having coronary artery disease (CAD) and heart failure, exhibiting relatively high prognostic impact and a 2.5 -fold increased incidence of heart failure compared to general population $[4,5]$, as well as an $8 \%$ increase in the risk of getting heart failure when glycosylated hemoglobin (HbA1c) is increased by $1 \%$ [6]

(c) The Author(s). 2018 Open Access This article is distributed under the terms of the Creative Commons Attribution 4.0 International License (http://creativecommons.org/licenses/by/4.0/), which permits unrestricted use, distribution, and reproduction in any medium, provided you give appropriate credit to the original author(s) and the source, provide a link to the Creative Commons license, and indicate if changes were made. The Creative Commons Public Domain Dedication waiver (http://creativecommons.org/publicdomain/zero/1.0/) applies to the data made available in this article, unless otherwise stated. 
Studies have demonstrated that ${ }^{18}$ F-FDG PET/CT imaging was able to show glucose metabolism in various organs/tissues in diabetic patients, mainly because that the levels of myocardial FDG metabolism were closely related to the expression of glucose transmembrane transporter (GLUT-4) [7] and the changes in left ventricular functions [8]. However, all the imaging processes in previous studies used dynamic PET scan after the standardization of myocardial substrates metabolism environment (euglycemic-hyperinsulinemic clamp). And thereby the imaging procedure was complicated and time-consuming [9]. Currently, the most widely used imaging technique in clinic is the fasting static whole-body PET/CT scan, which is mainly used for diagnosing and staging malignancy, and also has an important role in evaluating ischemic heart disease. Although the non-specific ${ }^{18} \mathrm{~F}$-FDG uptake of myocardium in wholebody ${ }^{18} \mathrm{~F}$-FDG PET/CT is a common problem and can affect the result, the study from Lee YH et al. [10] showed that the myocardial FDG uptake was significantly correlated with cardiac diastolic function. Therefore, this method may have potential advantages in observing myocardial glucose metabolism in diabetic patients. In this study, we reviewed the fasting static whole-body PET/CT images of 191 subjects from our medical examination center, and analyzed the differences in myocardial FDG uptake among diabetic (DM) patients, impaired fasting glucose (IFG) group, and normal fasting glucose (NFG) group, in order to explore the clinical and metabolic factors affecting myocardial FDG uptake. Also, we explored the feasibility of using static whole-body PET/CT as a potential monitoring and follow-up tool for diabetic cardiomyopathy.

\section{Methods}

\section{Study subject}

We did retrospective analysis on the subjects who required ${ }^{18}$ F-FDG PET/CT examination at the medical examination center of our hospital from October 2010 to December 2015. Subjects with the following diseases were excluded from our study: acute liver/kidney dysfunction, coronary heart disease or heart failure within the past 6 months, history of myocardial infarction, dilated/hypertrophic cardiomyopathy, history of malignancy, insulin-dependent diabetes, oral administration of steroid hormones/biologics, vasculitis, collagen diseases, acute and chronic infections. A total of 191 subjects were enrolled in this study. Their gender, age, height, weight, body mass index (BMI), smoking habit (excluding passive smoking) and drinking habit (at least once a week) were recorded. The flowchart of our study protocol is shown in Fig. 1. The study protocol conformed to the tenets of Declaration of Helsinki and was approved by the ethics committee of the third affiliated hospital of Soochow university.

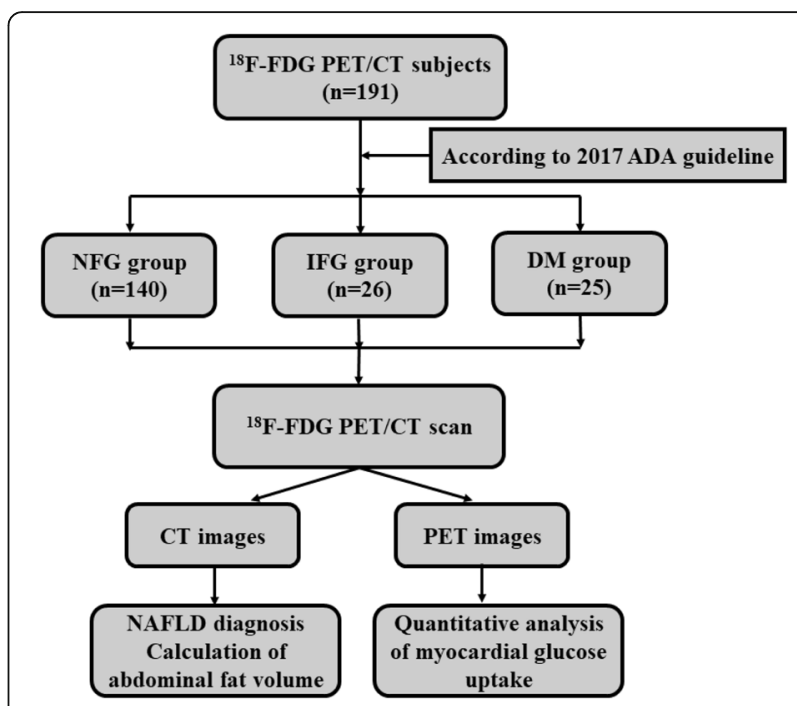

Fig. 1 The flowcharts of subjects grouping, PET/CT scanning and image analysis. ADA: American Diabetes Association, NFG: normal fasting glucose, IFG: impaired fasting glucose, DM: diabetes mellitus, NAFLD: nonalcoholic fatty liver disease

\section{Measurement of laboratory parameters}

The blood samples of the subjects (fasting venous blood $5 \mathrm{~mL}$, subjects were fasted for at least $8 \mathrm{~h}$ ) were collected before ${ }^{18} \mathrm{~F}$-FDG PET/CT scan. Enzymatic methods were used to measure liver functions (Alanine transaminase (ALT), glutamic oxalacetic transaminase (AST), glutamyltranspeptidase $(\gamma-\mathrm{GT})$ ), blood lipid (cholesterol (TC), triglyceride (TG), high density lipoprotein cholesterol (HDL-C), low density lipoprotein cholesterol (LDL-C), Lipoprotein (a)), and fasting blood glucose. The instrument we used was Hitachi 7600-120 automatic biochemical analyzer. The electrochemiluminescence method was used to determine the levels of thyroid stimulating hormone (TSH) and fasting insulin, conducted by Roche Cobas 8000 automatic electrochemical immunoanalyzer. High pressure liquid chromatography was used to measure fasting glycosylated hemoglobin (HbA1c), performed on BIO-RAD D-10 HbA1c meter. According to the criteria for medical diagnosis and treatment of diabetes, which were formulated by American Diabetes Association in 2017 [11], the patients with fasting glucose $\geq 7.0 \mathrm{mmol} / \mathrm{L}$, or $\mathrm{HbA} 1 \mathrm{c} \geq$ $6.5 \%$, or previously diagnosed as diabetes were all classified as DM group; the patients with fasting blood glucose ranging between $6.1 \mathrm{mmol} / \mathrm{L}$ and $7.0 \mathrm{mmol} / \mathrm{L}$ were classified as IFG group; others were classified as NFG group.

\section{PET/CT scan}

The imaging machine was the Biograph mCT (64) PET/ CT scan machine from German Siemens, and ${ }^{18}$ F-FDG (radiochemical purity > 95\%) was used as imaging agent. The subjects were fasted for more than $8 \mathrm{~h}$ before scan. After measuring fasting blood glucose, the patients were 
intravenously injected with ${ }^{18} \mathrm{~F}$-FDG (mean dose was 4.51 $\pm 0.77 \mathrm{MBq} / \mathrm{kg}$ ), and placed in a quiet, warm and dark place to rest for $45 \sim 60 \mathrm{~min}$. After urination, the patients were subjected to PET/CT scan. CT scan used the CareDose 4D technology (the tube current during CT scan is automatically adjusted according to patient's body size, anatomy and tissue density), with tube voltage $100 \mathrm{kV}$, screw pitch 0.8 , rotation time of bulb tube monolayer $0.5 \mathrm{~s}$, layer thickness $5 \mathrm{~mm}$, and reference milliampere seconds $60 \sim 180 \mathrm{mAs}$. PET scan was conducted immediately after CT scan with 3D mode. The scanning area ranged from skull base to upper femur, and acquisition time was $2 \mathrm{~min} / \mathrm{bed}$. Syngo TureD system was used to reconstruct images, forming cross-sectional, coronal and sagittal tomographic images, and three-dimensional projection images.

\section{CT diagnosis of nonalcoholic fatty liver disease (NAFLD)} The diagnosis of NAFLD was only based on CT images. Four regions of interest with a diameter of $4 \mathrm{~cm}$ were drawn on the left liver lobe, right anterior liver lobe, upper segment and lower segment of right posterior liver lobe, respectively. Two regions of interest with a diameter of $2 \mathrm{~cm}$ were drawn on the spleen (the two ROIs were $1.5 \mathrm{~cm}$ apart in depth) [12]. The average CT values of liver and spleen were calculated. If the CT value spleen $/ C T$ value liver was greater than 1.1, and the subject had no prior history of alcohol consumption, then this subject was diagnosed as NAFLD [13]. The selected ROIs did not include biliary structures, major arterial and venous vessels, and the area of the hepatic cyst.

\section{Calculation of abdominal fat volume}

Abdominal fat volume was divided into visceral adipose tissue (VAT) volume and subcutaneous adipose tissue (SAT) volume. The body CT image was reconstructed to $5 \mathrm{~mm}$ thickness. By using CT volume quantification software, VAT and SAT were sketched layer by layer from the S1 vertebra body up to abdominal muscle (25 continuous layers, $125 \mathrm{~mm}$ in total) [14]. Fat was defined as any voxel between -190 to $-60 \mathrm{HU}$. VAT and SAT volumes were automatically calculated and recorded.

\section{Quantitative analysis of myocardial glucose uptake}

First, two experienced nuclear medicine physicians were asked to categorize myocardial glucose uptake into four grades based on visual evaluation [15]: grade $0=$ minimal uptake, grade $1=$ mostly minimal or mild uptake, grade $2=$ mostly intense or moderate uptake, and grade $3=$ homogeneously intense uptake (Fig. 2). When the grading was inconsistent between the two physicians, it was determined by mutual consultation. Next, the regions of interest on left ventricle were sketched layer by layer on the PET/CT combined images, and Siemens TrueD software was used to automatically calculate the averaged
${ }^{18}$ F-FDG standard uptake of the entire left ventricle (SUVmean). When the subject had low myocardial uptake as a blood pool, an ROI consisting of double U-shaped lines with about $1 \mathrm{~cm}$ distance was drawn along the border of the left ventricle on the transaxial PET/CT images $[16,17]$.

\section{Statistical analysis}

SPSS 23.0 statistical software was used for statistical analysis. The measurement data were first tested by Kolmogorov-Smirnov test to check its normality. The normally distributed data was expressed as mean \pm standard deviation, and the non-normally distributed data was expressed as median (P25, P75). One-way ANOVA was used to compare the mean values among multiple groups with normal distribution, and Kruskal-Wallis test was used to compare the median of non-normally distributed groups. The counting data was expressed in terms of frequency and percentage. $\chi^{2}$ test was used to compare ratios between groups. When any theoretical frequencies in the list were less than 1, the Fisher exact test was conducted. ROC curve analysis was used to assess the efficacy of using visual grading of myocardial glucose uptake to predict diabetes. Spearman correlation analysis was used to analyze the correlations between visual grading of myocardial glucose uptake and its possible influencing factors. The independent influencing factors of myocardial glucose uptake grade were analyzed by multivariate Logistic regression, and the regression coefficients, Odds Ratio (OR) and 95\% confidence intervals were calculated. $P$ values were calculated by two-sided test. $P<0.05$ was considered statistically significant.

\section{Results}

Baseline characteristics of study subjects

A total of 191 subjects were included in the study, with an averaged age of $48.8 \pm 9.0$ years old. $67.5 \%(129 / 191)$ of the subjects were male, with the mean BMI of $24.8 \pm$ $2.9 \mathrm{~kg} / \mathrm{m}^{2}$. Among them, 40.8\% (78/191) were smokers, $36.6 \%$ (70/191) were drinkers, $13.6 \%$ (26/191) had impaired fasting glucose, $13.1 \%(25 / 191)$ had diabetes, and $17.3 \%$ (30/191) had NAFLD. The characteristics of NFG, IFG and DM groups, as well as the comparisons are shown in Table 1. Compared to NFG and IFG groups, DM group showed significant cardiovascular-related metabolic disorders, including overweight/obesity (significant increase in BMI and VAT), high NAFLD proportion, abnormal liver functions (elevated ALT and $\gamma$-GT levels), abnormal glucose metabolism (increased fasting blood glucose, HbA1c and fasting insulin), abnormal lipid metabolism (elevated TC, TG, LDL-C, and TC/ HDL-C levels, decreased HDL-C level), and insulin resistance (increased HOMA-IR) $(P<0.05$ for all). 


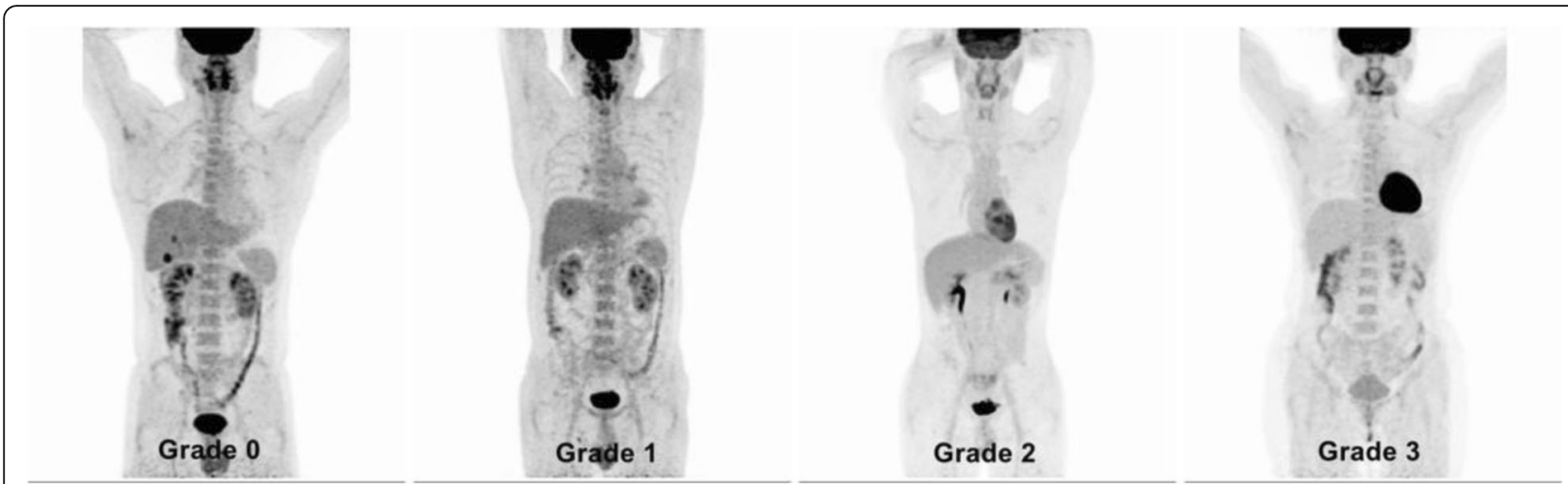

Fig. 2 Visual grading scale of myocardial FDG uptake. $\left({ }^{18} \mathrm{~F}-\mathrm{FDG} \mathrm{PET} / \mathrm{CT}\right.$ maximal intensity projection images)

\section{Correlation between myocardial glucose uptake and diabetes}

As shown in Fig. 2, the myocardial FDG uptake was divided into 4 grades based on visual evaluation. Among the 191 subjects, grade $0,1,2,3$ accounted for $24.1 \%$, $23.0 \%, 32.5 \%$ and $20.4 \%$ of the patients, respectively, and the corresponding SUVmean were $1.29 \pm 0.21,1.95$ $\pm 0.47,3.60 \pm 0.70$ and $5.97 \pm 1.25$, which were significantly different among all groups $(\mathrm{F}=338.14, P<0.001$, Fig. 3). The visual grading of myocardial FDG uptake was positively correlated with SUVmean $(r=0.941, P<$ $0.001)$.

Table 1 Baseline characteristics of study subjects

\begin{tabular}{|c|c|c|c|c|}
\hline Parameters & $\operatorname{NFG}(n=140)$ & $\mathrm{IFG}(n=26)$ & Diabetes $(n=25)$ & $P$ value \\
\hline Male (\%) & 65.0 & 65.4 & 84.0 & 0.169 \\
\hline Age (years) & $48.0 \pm 9.0$ & $51.4 \pm 9.1$ & $50.2 \pm 8.3$ & 0.143 \\
\hline BMI $\left(\mathrm{kg} / \mathrm{m}^{2}\right)$ & $24.6 \pm 2.9$ & $24.5 \pm 2.2$ & $26.6 \pm 3.2$ & $0.004^{*}$ \\
\hline Current smokers (\%) & 37.9 & 42.3 & 56.0 & 0.233 \\
\hline Current drinkers (\%) & 35.7 & 34.6 & 44.0 & 0.711 \\
\hline $\mathrm{ALT}(\mathrm{u} / \mathrm{L})$ & $24.0(16.3,32.0)$ & $25.5(19.0,36.0)$ & $28.9(21.5,54.5)$ & $0.020^{* \#}$ \\
\hline AST (u/L) & $18.9 \pm 4.7$ & $19.7 \pm 6.7$ & $21.3 \pm 8.1$ & 0.128 \\
\hline$\gamma-G T(u / L)$ & $27.0(19.0,47.8)$ & $36.0(20.5,70.8)$ & $46.0(32.0,90.0)$ & $<0.001^{* \#}$ \\
\hline TC (mmol/ L) & $4.95 \pm 0.95$ & $5.11 \pm 1.09$ & $5.59 \pm 1.28$ & $0.015^{*}$ \\
\hline TG (mmol/ L) & $2.16(1.52,2.96)$ & $2.51(1.87,3.67)$ & $3.20(2.39,5.83)$ & $0.001^{* \#}$ \\
\hline $\mathrm{HDL}-\mathrm{C}(\mathrm{mmol} / \mathrm{L})$ & $1.19 \pm 0.27$ & $1.23 \pm 0.33$ & $0.99 \pm 0.19$ & $0.002^{*}$ \\
\hline LDL-C (mmol/ L) & $2.44 \pm 0.60$ & $2.43 \pm 0.56$ & $2.86 \pm 0.83$ & $0.009^{*}$ \\
\hline TC/HDL-C & $4.36 \pm 1.29$ & $4.41 \pm 1.39$ & $5.80 \pm 1.58$ & $<0.001^{*}$ \\
\hline Lipoprotein (a) (mg/L) & $63.0(44.0,115.0)$ & $87.0(44.0,151.8)$ & $66.0(42.5,126.5)$ & $0.518^{\#}$ \\
\hline TSH ( $\mu \mathrm{IU} / \mathrm{ml})$ & $2.09(1.51,3.23)$ & $2.03(1.22,2.60)$ & $2.33(1.58,2.72)$ & $0.454^{\#}$ \\
\hline Fasting glucose (mmol/L) & $5.31 \pm 0.42$ & $6.40 \pm 0.19$ & $8.18 \pm 1.61$ & $<0.001^{*}$ \\
\hline HbA1c (\%) & $5.35 \pm 0.35$ & $5.76 \pm 0.50$ & $7.04 \pm 1.35$ & $<0.001^{*}$ \\
\hline Fasting insulin (mIU/L) & $5.98(4.44,8.11)$ & $5.63(5.10,7.99)$ & $9.13(5.68,13.24)$ & $0.001^{* \#}$ \\
\hline HOMA-IR & $1.39(1.01,1.98)$ & $1.56(1.42,2.31)$ & $3.33(2.42,4.68)$ & $<0.001^{\text {*\# }}$ \\
\hline NAFLD (\%) & 14.3 & 15.4 & 36.0 & $0.029^{*}$ \\
\hline VAT volume $\left(\mathrm{cm}^{3}\right)$ & $1281(875,1709)$ & $1452(1114,1714)$ & $1699(1364,2068)$ & $0.002^{* \#}$ \\
\hline SAT volume $\left(\mathrm{cm}^{3}\right)$ & $1778(1491,2217)$ & $1736(1330,2343)$ & $1714(1452,2277)$ & $0.999^{\#}$ \\
\hline
\end{tabular}

BMI: body mass index, ALT: glutamic-pyruvic transaminase, AST: glutamic-oxalacetic transaminease, $\gamma$-GT: glutamyl transpeptidase, TC: total cholesterol, TG: triglyceride, $H D L-C$ : high density lipoprotein cholesterol, $L D L-C$ : low density lipoprotein cholesterol, NAFLD: nonalcoholic fatty liver disease, VAT: visceral adipose tissue, SAT: subcutaneous adipose tissue

HOMA-IR = fasting glucosexfasting insulin /22.5

* indicates statistically siegnificant difference, \# indicates Kruskal-Wallis test. 


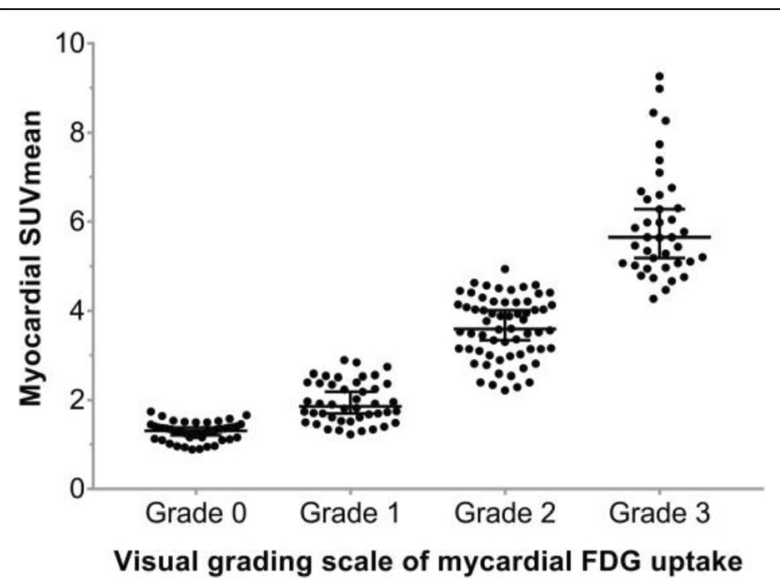

Fig. 3 Myocardial SUVmean for each visual grade. SUVmean of grade $0-3$ is $1.29 \pm 0.21,1.95 \pm 0.47,3.60 \pm 0.70,5.97 \pm 1.25$, respectively. $(F=338.14, P<0.001)$

The SUVmean of NFG, IFG and DM groups were $3.42 \pm$ $1.92,2.86 \pm 1.56$ and $1.94 \pm 0.96$, respectively, significantly different among the three groups $(\mathrm{F}=7.69, P=0.01$, Fig. 4b). Comparisons between any two groups showed that, the SUVmean of DM group was significantly lower than IFG and NFG groups $(P<0.05)$; the SUVmean of IFG group was lower than NFG group, but not significantly $(P$ $=0.312$ ). Moreover, in the DM group, up to $48.0 \%$ showed myocardial FDG uptake in grade $0,36.0 \%$ were in grade 1 , and none of them was in grade 3 (Fig. 4a). The ROC curve analysis of myocardial FDG uptake was used to predict the incidence of diabetes. When myocardial FDG uptake $\leq$ Grade 1, the prediction sensitivity was $84.0 \%$, and the specificity was $58.4 \%$, with AUC of 0.745 , and $95 \%$ confidence interval of $0.677 \sim 0.805(\mathrm{z}=5.859, P<0.001$, Fig. 5).

\section{The effects of cardiovascular-related metabolic disorders on myocardial FDG uptake}

The correlations between visual grading of FDG uptake and the subjects' general characteristics, as well as their metabolic parameters were analyzed by univariate analysis (Table 2). The analysis showed that myocardial FDG uptake had significant positive correlation with HDL-C, and significant negative correlations with BMI, smoking, drinking, ALT, $\gamma$-GT, TG, TC/HDL-C, fasting blood glucose, HbA1c, fasting insulin, HOMA-IR, NAFLD ratio, and VAT volume. The correlation coefficients are shown in Table 2. According to the ROC curve, grade 0 and grade 1 were defined as poor FDG uptake, and grade 2 and grade 3 were defined as good FDG uptake. Based on these two categories, the multivariate Logistic stepwise regression analysis showed that gender (male), HOMA-IR and NAFLD were independent risk factors for poor myocardial FDG uptake (OR > $1, P<0.01$, Table 3$)$.

\section{Discussion}

In this study, we found that visual grading of myocardial FDG uptake had a very good positive correlation with myocardial SUVmean $(r=0.941, P<0.001)$, thereby it can reflect the levels of fasting myocardial glucose metabolism. Under physiological conditions, FFA and glucose oxidation are the major sources of myocardial energy. The process of substrate selection is dynamic and largely depends on substrate availability, oxygen concentration, and myocardial workload [1]. Studies have shown that, in static whole-body PET/CT scan, the myocardial glucose usage during fasting is largely variable, which indirectly reflects the flexibility of myocardial substrate utilization [15, 18]. Among the 191 subjects in our study, the myocardial SUVmean ranged from 0.89 to 9.26 , and subjects with visual grade $0-3$ accounted for $24.1 \%, 23.0 \%, 32.5 \%$ and $20.4 \%$, respectively. These results were consistent with the previous studies.

Myocardial energy metabolism disorders in diabetes is an important cause of $\mathrm{CAD}$ and heart failure [1, 5, 19]. Long-term abnormal glucose and lipid metabolisms, as well as insulin resistance, can lead to cardiac microvascular endothelial cell proliferation, basement membrane thickening, oxygen utilization reduction, degeneration of heart myofibers and perivascular fibers, and deposition of intracoronary and intramyocardial glycoproteins, collagen fibers, triglycerides and cholesterol, all of which can result in coronary artery lumen stenosis, myocardial energy metabolism disorders, decreased systolic/diastolic function, and eventually lead to CAD and heart failure. In this study, we found that DM group had multiple cardiovascular metabolic disorders, such as overweight/obesity, abnormal glucose and lipid metabolisms, NAFLD, and insulin resistance. Moreover, up to $84 \%$ of DM group showed poor FDG uptake (Grade 0/1), decreased variation range of SUVmean, and significantly lower myocardial SUVmean, as compared to IFG and NFG groups (Fig. 4b). These results indicate that in diabetes, the myocardial glucose metabolism and the flexibility of substrate utilization are both reduced.

When using myocardial FDG uptake less or equal to grade 1 to predict diabetes, the sensitivity was $84.0 \%$, but the specificity was only $58.4 \%$, suggesting that diabetes is not the only factor that leads to reduced myocardial glucose metabolism. Furthermore, the univariate analysis indicates that multiple metabolic factors can cause decreased myocardial glucose metabolism, which is consistent with the study from Kim G et al. [20]. However, their study used the ratio of heart SUV to liver SUV to reflect the myocardial FDG uptake, and it is still questionable that whether these two SUV values are collinear in insulin resistance (in our preliminary study, we found a weak correlation between heart SUVmean and liver SUVmean, $r=-0.151, P=0.037)$; meanwhile, their study did not describe the percentage of NAFLD patients in study population, but we found NAFLD, gender (male), 

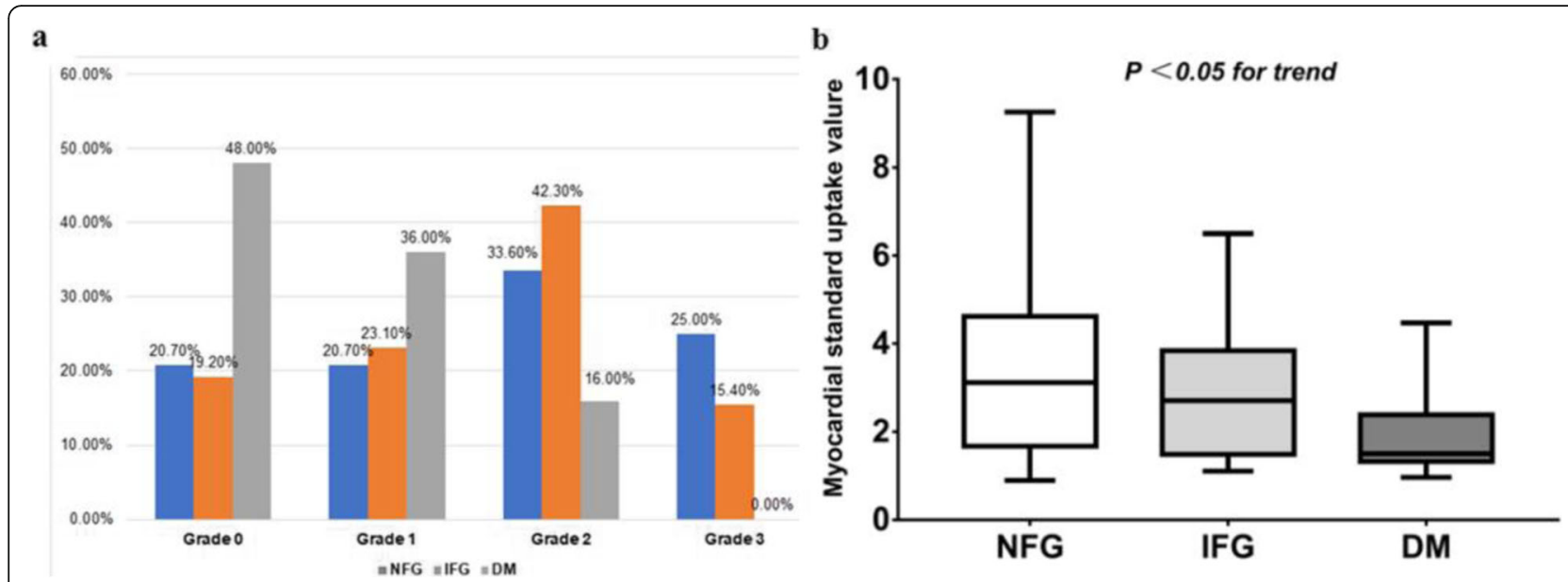

Fig. 4 Correlation between myocardial glucose uptake and diabetes. $\mathbf{a}$, the distribution of NFG, IFG and DM groups in each myocardial glucose uptake grade; $\mathbf{b}$, the differences in SUVmean between NFG, IFG and DM groups (NFG: $3.42 \pm 1.92$, IFG: $2.86 \pm 1.56$, DM: $1.94 \pm 0.96, P<0.05$ )

HOMA-IR are independent risk factors for poor myocardial FDG uptake.

Steady-state insulin assessment model (HOMA-IR) can reflect the degree of whole body insulin resistance during fasting. Since myocardium is one of the insulin target organs, when myocardium is insulin resistant, myocardial FDG uptake will decrease. Our study shows that HOMA-IR is an independent risk factor for poor myocardial FDG uptake $(\mathrm{OR}=1.755, P=0.003)$. It has been shown [21] that the major factors contributing to cardiac insulin resistance are oxidative stress, hyperglycemia, hyperlipidemia, dysregulated secretion of adipokines/cytokines, and

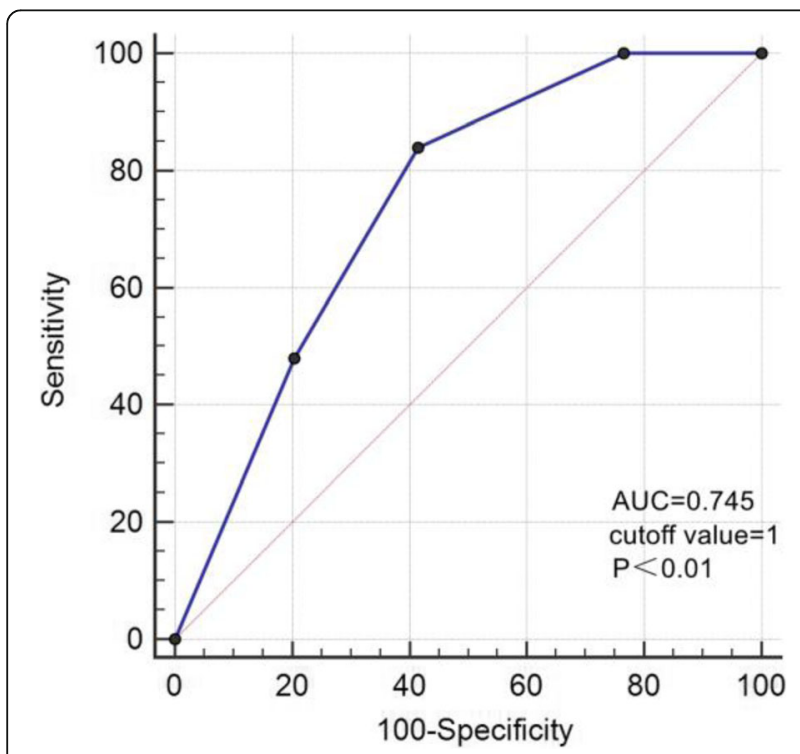

Fig. 5 ROC curve analysis was used to assess the efficacy of using visual grading of myocardial FDG uptake to predict diabetes. When FDG uptake $\leq$ Grade 1, the sensitivity of predicting diabetes was $84 \%$, the specificity was $58.43 \%, A \cup C=0.745$, and $95 \%$ confidence interval was $0.677 \sim 0.805(z=5.859, P<0.001)$ inappropriate activation of renin-angiotensin II-aldosterone system (RAAS) and sympathetic nervous system. When glucose oxidation is decreased in cardiomyocytes, the fatty acids uptake and lipid metabolism are enhanced, which stimulates the tricarboxylic acid (TCA) cycle. The increased TCA cycle elevates citric acid levels, inhibits phosphofructokinase activity, reduces glycolysis rate, and activates the phosphorylation of pyruvate dehydrogenase, which then leads to inhibited pyruvate complex. All of these changes can result in increased acidosis in cardiomyocytes, sarcoplasmic reticulum swelling, and $\mathrm{Ca}^{2}$ influx blockage. During acidosis, $\mathrm{H}^{+}$and $\mathrm{Ca}^{2+}$ competitively bind to myosin, causing the failure of excitement-contraction coupling and eventually leading to reduced myocardial contractility [22].

When the volume of fat tissue, which is responsible for energy storage, is saturated, excessive fat is ectopically deposited in liver, causing lipotoxicity and insulin resistance [23]. NAFLD is the manifestation of metabolic syndrome in liver, and is also an important risk factor for cardiovascular disease. Recent studies have reported that NAFLD is associated with altered high-energy phosphate metabolism [24], and subclinical myocardial remodeling and dysfunction [25]. Interestingly, in our study, we found NAFLD was also an independent risk factor for poor FDG uptake in myocardium $(\mathrm{OR}=3.082, P=0.023)$, suggesting that NAFLD is also involved in the energy substrate metabolism of myocardium. Several large population-based cohort studies have demonstrated that moderately elevated GGT levels, which are potential markers for NAFLD and atherosclerosis, are independently associated with increased risk of heart failure [26]. Therefore, our study provides further evidence to demonstrate the possible link between NAFLD and heart failure.

The current study also exhibits several limitations. Since this study was a retrospective study, none of the subjects underwent oral glucose tolerance test. Thereby 
Table 2 Correlation between visual grading of myocardial FDG uptake and metabolic factors

\begin{tabular}{|c|c|c|c|c|c|c|}
\hline Parameters & Grade $0(n=46)$ & Grade $1(n=44)$ & Grade $2(n=62)$ & Grade $3(n=39)$ & r & $P$ value \\
\hline Male (\%) & 89.1 & 72.7 & 61.3 & 46.2 & -0.319 & $<0.001^{*}$ \\
\hline Age (years) & $48.0 \pm 7.9$ & $50.7 \pm 9.7$ & $49.8 \pm 9.2$ & $48.8 \pm 9.0$ & 0.107 & 0.139 \\
\hline BMI $\left(\mathrm{kg} / \mathrm{m}^{2}\right)$ & $25.6 \pm 3.1$ & $25.3 \pm 2.9$ & $24.3 \pm 2.7$ & $24.1 \pm 2.9$ & -0.176 & $0.015^{*}$ \\
\hline Current smokers (\%) & 34.6 & 24.4 & 26.9 & 14.1 & -0.222 & $0.002^{*}$ \\
\hline Current drinkers (\%) & 27.1 & 31.4 & 28.6 & 12.9 & -0.161 & $0.026^{*}$ \\
\hline $\operatorname{ALT}(\mathrm{u} / \mathrm{L})$ & $30.5(19.8,42.0)$ & $25.5(21.0,35.8)$ & $22.0(18.0,30.3)$ & $19.0(14.0,29.0)$ & -0.281 & $<0.001^{*}$ \\
\hline AST (u/L) & $20.8 \pm 7.0$ & $19.6 \pm 6.0$ & $18.8 \pm 4.4$ & $18.1 \pm 4.6$ & -0.127 & 0.080 \\
\hline Y-GT (u/L) & $44.0(27.8,72.8)$ & $34.0(23.3,68.5)$ & $25.0(18.0,43.8)$ & $24.0(15.0,37.0)$ & -0.345 & $<0.001^{*}$ \\
\hline TC (mmol/ L) & $5.20 \pm 1.36$ & $5.10 \pm 0.85$ & $4.84 \pm 0.81$ & $5.17 \pm 1.09$ & -0.026 & 0.719 \\
\hline TG (mmol/ L) & $2.78(1.77,4.01)$ & $2.53(1.78,3.81)$ & $2.11(1.45,2.67)$ & $2.03(1.52,2.71)$ & -0.231 & $0.001^{*}$ \\
\hline $\mathrm{HDL}-\mathrm{C}(\mathrm{mmol} / \mathrm{L})$ & $1.05 \pm 0.27$ & $1.09 \pm 0.20$ & $1.24 \pm 0.28$ & $1.27 \pm 0.30$ & 0.326 & $<0.001^{*}$ \\
\hline LDL-C (mmol/ L) & $2.60 \pm 0.84$ & $2.53 \pm 0.54$ & $2.36 \pm 0.51$ & $2.55 \pm 0.67$ & -0.042 & 0.569 \\
\hline $\mathrm{TC} / \mathrm{HDL}-\mathrm{C}$ & $5.21 \pm 1.79$ & $4.81 \pm 1.19$ & $4.09 \pm 1.16$ & $4.22 \pm 1.24$ & -0.291 & $<0.001^{*}$ \\
\hline Lipoprotein(a) (mg/L) & $61.5(37.8,131.0)$ & $64.0(49.0,125.0)$ & $65.5(44.8,116.5)$ & $73.0(37.0,122.0)$ & -0.006 & 0.934 \\
\hline TSH $(\mu \mathrm{l} U / \mathrm{ml})$ & $2.02(1.44,2.83)$ & $2.03(1.47,2.64)$ & $2.16(1.56,3.53)$ & $2.16(1.48,3.25)$ & 0.093 & 0.201 \\
\hline Fasting glucose (mmol/ L) & $6.28 \pm 1.57$ & $5.97 \pm 1.16$ & $5.64 \pm 1.10$ & $5.45 \pm 0.51$ & -0.219 & $0.002^{*}$ \\
\hline $\mathrm{HbA1c( \% )}$ & $5.85 \pm 1.01$ & $5.81 \pm 0.98$ & $5.45 \pm 0.69$ & $5.45 \pm 0.44$ & -0.173 & $0.018^{*}$ \\
\hline Fasting insulin (m/U/L) & $7.90(5.46,10.40)$ & $7.40(4.86,9.92)$ & $5.70(4.51,6.78)$ & $5.20(4.00,6.89)$ & -0.322 & $<0.001^{*}$ \\
\hline HOMA-IR & $2.17(1.38,2.88)$ & $1.91(1.36,2.47)$ & $1.29(1.06,1.63)$ & $1.37(0.95,1.60)$ & -0.365 & $<0.001^{*}$ \\
\hline NAFLD (\%) & 54.5 & 24.2 & 18.2 & 3.0 & -0.337 & $<0.001^{*}$ \\
\hline VAT volume $\left(\mathrm{cm}^{3}\right)$ & $1462(1214,1833)$ & $1571(1042,1900)$ & $1339(950,1731)$ & $1084(766,1450)$ & -0.239 & $0.001^{*}$ \\
\hline SAT volume $\left(\mathrm{cm}^{3}\right)$ & $1693(1354,2021)$ & $1712(1396,2190)$ & $1781(1516,2183)$ & $1841(1490,2375)$ & 0.116 & 0.109 \\
\hline
\end{tabular}

BMI: body mass index, ALT: glutamic-pyruvic transaminase, AST: glutamic-oxalacetic transaminease, $\gamma$-GT: glutamyl transpeptidase, TC: total cholesterol, TG: triglyceride,HDL-C: high density lipoprotein cholesterol, LDL-C: low density lipoprotein cholesterol, NAFLD: nonalcoholic fatty liver disease, VAT: visceral adipose tissue, SAT: subcutaneous adipose tissue

HOMA-IR = fasting glucosexfasting insulin /22.5

* indicates statistically significant difference.

some subjects in the NFG group might have impaired glucose tolerance (IGT), which may affect the differences between IFG and NFG group (IFG and IGT both belong to pre-diabetes). In addition, the study subjects were free of cardiovascular disease, and the diabetes group only accounted for $13.1 \%$. Although the diabetes ratio was consistent with the diabetes incidence in local population [27], the number was relatively small, thereby we couldn't investigate the influencing factors of myocardial glucose metabolism in diabetic patients.

Table 3 Logistic regression analysis of factors affecting myocardial FDG uptake

\begin{tabular}{lllll}
\hline Variables & Regression coefficient & OR & $95 \% \mathrm{Cl}$ & $P$ value \\
\hline Male & 1.045 & 2.844 & $1.390 \sim 5.816$ & 0.004 \\
HOMA-IR & 0.562 & 1.755 & $1.207 \sim 2.511$ & 0.003 \\
NAFLD & 1.126 & 3.082 & $1.171 \sim 8.111$ & 0.023 \\
\hline
\end{tabular}

NAFLD: nonalcoholic fatty liver disease HOMA-IR = fasting glucosexfasting insulin /22.5

\section{Conclusions}

In summary, static whole-body PET/CT is a simple and easy method to study myocardial glucose metabolism. The visual grading of myocardial FDG uptake had highly positive correlation with myocardial SUVmean. Diabetes is significantly associated with cardiovascular-related metabolic disorders and reduced myocardial glucose metabolism. Using decreased myocardial glucose metabolism to predict diabetes is highly sensitive, but with a relatively low specificity (only 58.4\%), suggesting that diabetes is not the only factor causing reduced myocardial glucose metabolism. Univariate analysis demonstrated that multiple factors (gender, overweight/obesity, lifestyle, laboratory parameters, and NAFLD) could lead to decreased myocardial glucose metabolism. Moreover, multivariate regression analysis showed that gender (male), HOMA-IR and NAFLD were independent risk factors for poor myocardial FDG uptake.

\section{Abbreviations}

DM: Diabetes mellitus; FDG: Fluorodeoxyglucose; HDL: High density lipoprotein; HF: Heart failure; LDL: Low density lipoprotein; MBq: Megabecquerel; 
NAFLD: Nonalcoholic fatty liver disease; PET: Positron emission tomography; ROI: Region of interest; TC: Total cholesterol; TG: Triglycerides

\section{Acknowledgements}

Not applicable.

\section{Funding}

This study was supported by National Natural Science Foundation of China (81471690); National Natural Science Foundation for Young Scientists (81701734); Science and Technology Plan of Jiangsu Province - standardization of diagnosis and treatment of key diseases project (BE2015635). None of these agencies were involved in designing, analyzing, or interpreting the study results.

\section{Availability of data and materials}

The datasets used and/or analysed during the current study are available from the corresponding author on reasonable request.

\section{Authors' contributions}

XLS and YTW conceived and designed the study. LJH drafted the manuscript. MX was responsible for PET/CT scan and image quality control. QC and XSW were responsible for data collection and image analysis. LJH, $\mathrm{XLS}$ and YTW performed the statistical analysis. All authors read and approved the final manuscript.

\section{Ethics approval and consent to participate}

The study was approved by the ethics committee of the Third Affiliated Hospital of Soochow University, and written informed consent was obtained from all patients.

\section{Consent for publication}

Not applicable.

\section{Competing interests}

The authors declare that they have no competing interests.

\section{Publisher's Note}

Springer Nature remains neutral with regard to jurisdictional claims in published maps and institutional affiliations.

\section{Author details}

'Department of Radiation Oncology, The Second People's Hospital of Changzhou, Nanjing Medical University, Changzhou 213003, Jiangsu, China. ${ }^{2}$ Department of Nuclear Medicine, The Third Affiliated Hospital of Soochow University, Changzhou 213003, Jiangsu, China.

\section{Received: 30 July 2018 Accepted: 19 October 2018}

\section{Published online: 29 October 2018}

\section{References}

1. Chong CR, Clarke K, Levelt E. Metabolic remodeling in diabetic cardiomyopathy. Cardiovasc Res. 2017;113:422-30.

2. Gropler RJ, Beanlands RS, Dilsizian V, Lewandowski ED, Villanueva FS, Ziadi MC. Imaging myocardial metabolic remodeling. J Nucl Med. 2010;51:88s-101s.

3. Low Wang CC, Hess CN, Hiatt WR, Clinical Update GAB. Cardiovascular disease in diabetes mellitus: atherosclerotic cardiovascular disease and heart failure in type 2 diabetes mellitus - mechanisms, management. and Clinical Considerations Circulation. 2016:133:2459-502.

4. Bahtiyar G, Gutterman D, Lebovitz H. Heart failure: a major cardiovascular complication of diabetes mellitus. Curr Diab Rep. 2016;16:116.

5. Barbero U, D'Ascenzo F, Nijhoff F, Moretti C, Biondi-Zoccai G, Mennuni M, et al. Assessing risk in patients with stable coronary disease: when should we intensify care and follow-up? Results from a meta-analysis of observational studies of the COURAGE and FAME era. Scientifica (Cairo). 2016;2016: 3769152.

6. Parry HM, Deshmukh H, Levin D, Van Zuydam N, Elder DH, Morris AD, et al. Both high and low hba1c predict incident heart failure in type 2 diabetes mellitus. Circ Heart Fail. 2015;8:236-42.

7. Shoghi Kl, Gropler RJ, Sharp T, Herrero P, Fettig N, Su Y, et al. Time course of alterations in myocardial glucose utilization in the zucker diabetic fatty rat with correlation to gene expression of glucose transporters: a small-animal pet investigation. J Nucl Med. 2008;49:1320-7.

8. van den Brom CE, Huisman MC, Vlasblom R, Boontje NM, Duijst S, Lubberink $M$, et al. Altered myocardial substrate metabolism is associated with myocardial dysfunction in early diabetic cardiomyopathy in rats: studies using positron emission tomography. Cardiovasc Diabetol. 2009;8:39.

9. Fallavollita JA, Luisi AJ Jr, Yun E, deKemp RA, Canty JM Jr. An abbreviated hyperinsulinemic-euglycemic clamp results in similar myocardial glucose utilization in both diabetic and non-diabetic patients with ischemic cardiomyopathy. J Nucl Cardiol. 2010;17:637-45.

10. Lee YH, Kim KJ, Yoo ME, Kim G, Yoon HJ, Jo K, et al. Association of nonalcoholic steatohepatitis with subclinical myocardial dysfunction in noncirrhotic patients. J Hepatol. 2018;68:764-72.

11. American Diabetes Association. Standards of medical care in diabetes-2017. Diabetes Care. 2017:40:S1-S135.

12. Ozguven $\mathrm{S}$, Ones T, Yilmaz $\mathrm{Y}$, Turoglu HT, Imeryuz N. The role of active brown adipose tissue in human metabolism. Eur J Nucl Med Mol Imaging. 2016;43:355-61.

13. Goceri E, Shah ZK, Layman R, Jiang X, Gurcan MN. Quantification of liver fat: a comprehensive review. Comput Biol Med. 2016;71:174-89.

14. Rosenquist KJ, Pedley A, Massaro JM, Therkelsen KE, Murabito JM, Hoffmann $U$, et al. Visceral and subcutaneous fat quality and cardiometabolic risk. JACC Cardiovasc Imaging. 2013;6:762-71.

15. Williams G, Kolodny GM. Suppression of myocardial 18F-FDG uptake by preparing patients with a high-fat, low-carbohydrate diet. AJR Am J Roentgenol. 2008;190:W151-6.

16. Lang RM, Bierig M, Devereux RB, Flachskampf FA, Foster E, Pellikka PA, et al. Recommendations for chamber quantification: a report from the American Society of Echocardiography's guidelines and standards committee and the chamber quantification writing group, developed in conjunction with the European Association of Echocardiography, a branch of the European Society of Cardiology. J Am Soc Echocardiogr. 2005;18:1440-63.

17. Jeong J, Kong E, Chun K, Cho I. The impact of energy substrates, hormone level and subject-related factors on physiologic myocardial (18)F-FDG uptake in normal humans. Nucl Med Mol Imaging. 2013:47:225-31.

18. Lee HY, Nam HY, Shin SK. Comparison of myocardial F-18 FDG uptake between overnight and non-overnight fasting in non-diabetic healthy subjects. Jpn J Radiol. 2015;33:385-91.

19. Jia G, Whaley-Connell A, Sowers JR. Diabetic cardiomyopathy: a hyperglycaemia- and insulin-resistance-induced heart disease. Diabetologia. 2018;61:21-8.

20. Kim G, Jo K, Kim KJ, Lee YH, Han E, Yoon HJ, et al. Visceral adiposity is associated with altered myocardial glucose uptake measured by (18)FDGPET in 346 subjects with normal glucose tolerance, prediabetes, and type 2 diabetes. Cardiovasc Diabetol. 2015;14:148.

21. Aroor AR, Mandavia $\mathrm{CH}$, Sowers JR. Insulin resistance and heart failure. Molecular mechanisms Heart Fail Clin. 2012;8:609-17.

22. How OJ, Aasum E, Severson DL, Chan WY, Essop MF, Larsen TS. Increased myocardial oxygen consumption reduces cardiac efficiency in diabetic mice. Diabetes. 2006:55:466-73.

23. Samuel VT, Petersen KF, Shulman GI. Lipid-induced insulin resistance: Unravelling the mechanism. Lancet. 2010;375:2267-77.

24. Perseghin G, Lattuada G, De Cobelli F, Esposito A, Belloni E, Ntali G, et al. Increased mediastinal fat and impaired left ventricular energy metabolism in young men with newly found fatty liver. Hepatology. 2008;47:51-8.

25. VanWagner LB, Wilcox JE, Colangelo LA, Lloyd-Jones DM, Carr JJ, Lima JA, et al. Association of nonalcoholic fatty liver disease with subclinical myocardial remodeling and dysfunction: a population-based study. Hepatology. 2015; 62:773-83.

26. Wannamethee SG, Whincup PH, Shaper AG, Lennon L, Sattar N. Gammaglutamyltransferase, hepatic enzymes, and risk of incident heart failure in older men. Arterioscler Thromb Vasc Biol. 2012;32:830-5.

27. Wang L, Gao P, Zhang M, Huang Z, Zhang D, Deng Q, et al. Prevalence and ethnic pattern of diabetes and Prediabetes in China in 2013. JAMA. 2017; 317:2515-23. 